\title{
DESIGN AND APPLICATION OF AN IMAGE-PROCESSING-BASED FUZZY AUTOPILOT FOR SMALL-BOAT APPROACHING MANEUVERS
}

\author{
Sin-Der Lee \\ Department of Transportation Science, National Taiwan Ocean University, Keelung, Taiwan, R.O.C, \\ leesd@mail.ntou.edu.tw \\ Ching-Yaw Tzeng \\ Department of Communications, Navigation and Control Engineering, National Taiwan Ocean University, Keelung, \\ Taiwan, R.O.C. \\ Young-Zehr Kehr \\ Department of Systems Engineering and Naval Architecture, National Taiwan Ocean University, Keelung, Taiwan, \\ R.O.C \\ Chih-Kai Kang \\ Department of Communications, Navigation and Control Engineering, National Taiwan Ocean University, Keelung, \\ Taiwan, R.O.C \\ Chi-Chun Huang \\ Department of Communications, Navigation and Control Engineering, National Taiwan Ocean University, Keelung, \\ Taiwan, R.O.C
}

Follow this and additional works at: https://jmstt.ntou.edu.tw/journal

Part of the Electrical and Computer Engineering Commons

\author{
Recommended Citation \\ Lee, Sin-Der; Tzeng, Ching-Yaw; Kehr, Young-Zehr; Kang, Chih-Kai; and Huang, Chi-Chun (2010) "DESIGN AND \\ APPLICATION OF AN IMAGE-PROCESSING-BASED FUZZY AUTOPILOT FOR SMALL-BOAT APPROACHING \\ MANEUVERS," Journal of Marine Science and Technology. Vol. 18: Iss. 4, Article 11. \\ DOI: $10.51400 / 2709-6998.1914$ \\ Available at: https://jmstt.ntou.edu.tw/journal/vol18/iss4/11 \\ This Research Article is brought to you for free and open access by Journal of Marine Science and Technology. It has been \\ accepted for inclusion in Journal of Marine Science and Technology by an authorized editor of Journal of Marine Science and \\ Technology.
}




\section{DESIGN AND APPLICATION OF AN IMAGE-PROCESSING-BASED FUZZY}

AUTOPILOT FOR SMALL-BOAT APPROACHING MANEUVERS

\section{Acknowledgements}

This study was supported by the National Science Council of the Republic of China under Grant No. NSC-95-2221-E019-074-MY3. 


\title{
DESIGN AND APPLICATION OF AN IMAGE-PROCESSING-BASED FUZZY AUTOPILOT FOR SMALL-BOAT APPROACHING MANEUVERS
}

\author{
Sin-Der Lee*, Ching-Yaw Tzeng**, Young-Zehr Kehr***, Chih-Kai Kang**, and \\ Chi-Chun Huang**
}

Key words: image processing, visual guidance, approaching maneuver, fuzzy autopilot.

\begin{abstract}
This paper presents an image-processing-based fuzzy autopilot scheme for accomplishing small-boat approaching maneuvers in a harbor environment. In the proposed approach, two canvas targets are arranged in cascade on the quayside to form a leading line. The targets are detected by a charge coupled device (CCD) camera mounted on the bow of the boat, and their geometric centers are computed by a Hue- and Saturation-based image-processing scheme. The autopilot system calculates the current heading deviation and tracking deviation angles of the boat by analyzing the displacements of the target centers relative to the CCD center line. These angles are then supplied to a fuzzy-logic-based control system to determine the rudder commands required to bring the boat back on course. During the approaching maneuver, the autopilot system estimates the distance between the boat and the quayside using a simple trigonometric relationship, and at a certain pre-defined distance, automatically switches the steering control system from an approach mode to a berthing control mode. The experimental results obtained using a small FRP boat confirm the ability of the autopilot system to accomplish the approaching maneuver and show that the estimated value of the boat-to-quayside distance deviates by approximately $10 \sim 20 \%$ from the exact value.
\end{abstract}

Paper submitted 12/05/08; revised 07/23/09; accepted 08/14/09; Author for correspondence: Sin-Der Lee (e-mail: leesd@mail.ntou.edu.tw).

*Department of Transportation Science, National Taiwan Ocean University, Keelung, Taiwan, R.O.C.

**Department of Communications, Navigation and Control Engineering, National Taiwan Ocean University, Keelung, Taiwan, R.O.C.

***Department of Systems Engineering and Naval Architecture, National Taiwan Ocean University, Keelung, Taiwan, R.O.C.

\section{INTRODUCTION}

The potential for utilizing automatic control theory to accomplish the heading control of automatically-steered bodies was first demonstrated using a simple PID controller as far back as the 1920's [5, 8]. Broadly speaking, autopilots control system can be categorized as either "model-based", "model-free" or "hybrid", depending on their mode of operation. Amongst the model-based schemes, the linear quadratic - Gaussian (LQG) autopilot presented by Holzhüter [2] and the $H_{\infty}$ control system developed by Morawski and Pomirski [6] are two of the most well known examples. Meanwhile, typical examples of model-free autopilot systems include the fuzzy control scheme proposed by Vaneck [13] and the artificial neural network (ANN) berthing system presented by Zhang et al. [14]. Finally, some of the best known examples of hybrid type autopilots include the internal model control (IMC)-ANN system presented by Tzeng and Lu [12] and the fuzzy-sliding mode control scheme proposed by Huang [3].

As the capabilities of computer vision systems have improved in recent years, the feasibility of utilizing imageprocessing techniques to facilitate vehicle guidance and control has attracted increasing interest. For example, Proctor et al. [7] developed an image processor featuring a rejective cascade filter and an extended Kalman filter to enable the autonomous flight of a glider fitted with a single vision sensor to a known fixed object. Suzuki et al. [9] presented a humanoriented information restructuring (HIR) system based on a single camera to assist drivers in executing parking maneuvers in real-world situations. Chao [1] developed a vision-based scheme for the parallel parking of a car-like mobile robot, in which a feasible steering path was determined by processing the omni-directional images acquired by on-board cameras and the necessary steering wheel corrections were instructed by a fuzzy controller.

In ship handling practice, the process of guiding a ship into a harbor can be divided into four distinct stages, namely approaching, stopping, turning and berthing. During the ap- 
proaching maneuver, the pilot guides the ship along a leading line defined by carefully selected visual landmarks, beacons or leading lights, and applies course corrections as required to compensate for the effects of the prevailing current, unexpected changes in the wind direction, obstacles, and so forth. Once the ship arrives at a safety range from the berthing wall, the pilot slows the engines, turns the ship and berths carefully at the quayside. The current study develops an image processing - based autopilot scheme for mimicking the actions of a human pilot in guiding the ship along the leading line and determining a suitable point at which to commence the berthing procedure. In the proposed approach, a CCD camera is used to acquire an image of two targets arranged in cascade on the berthing wall, and an image-processing scheme is then applied to locate their respective geometric centers. Treating the imaginary line passing through the center points of the two targets as a notional leading line, the autopilot calculates the current heading and tracking deviation angles of the ship and inputs these information to a fuzzy-based controller, which then instructs the necessary rudder movements required to bring the ship back toward the leading line. It is to be noted a similar image processing-based approaching maneuver study while using the IMC design method has been reported by Lee et al. [4]. This paper; however, adopts the fuzzy logic control method, which bears resemblance to human pilot's operating behaviors. The feasibility of the proposed approach is verified by performing a series of experimental trials using a small FRP boat in a real-world harbor environment.

\section{IMAGE-PROCESSING ALGORITHM AND VISUAL GUIDANCE STRATEGY}

\section{Image-Processing Algorithm}

As described above, the autopilot system developed in this study is based on the leading line concept used by manual pilots when guiding a ship toward the berthing region within the harbor. In the proposed approach, the leading line is formed by pre-arranging two canvas targets on the quayside, namely a rear green target measuring $145 \mathrm{~cm} * 100 \mathrm{~cm}$ and a front blue target measuring $100 \mathrm{~cm} * 175 \mathrm{~cm}$. The targets are acquired by a CCD camera mounted on the bow of the boat and the resulting image is transformed from the RGB (red, green and blue) color space into an equivalent HSV (hue, saturation and value) space which more closely resembles the human perception of color. The color space transformations are performed using the following correlations [10]:

$$
\begin{aligned}
& \left(\frac{G-B}{M A X-M I N}\right) / 6, \quad \text { if } R=M A X \\
H= & \left(2+\frac{B-R}{M A X-M I N}\right) / 6, \quad \text { if } G=M A X \\
& \left(4+\frac{R-G}{M A X-M I N}\right) / 6, \quad \text { if } B=M A X
\end{aligned}
$$

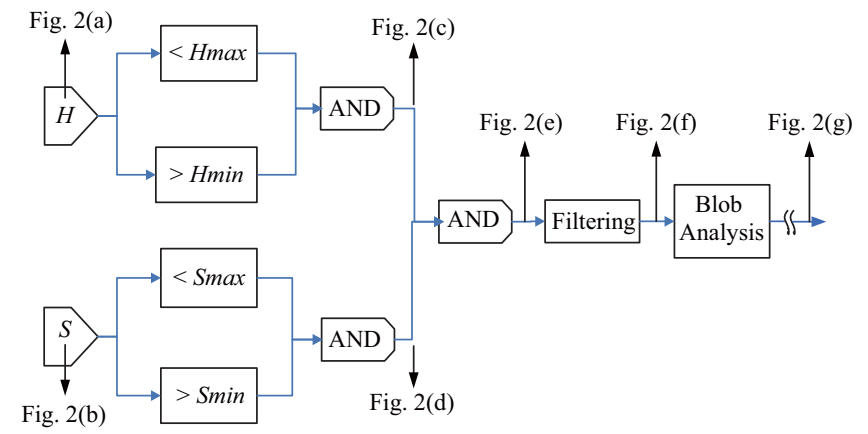

Fig. 1. Flowchart showing basic steps in image-processing scheme.

$$
\begin{gathered}
S=\frac{M A X-M I N}{M A X} \\
V=M A X
\end{gathered}
$$

where $H, S$ and $V$ are the hue, saturation and value, respectively, and $M A X$ and $M I N$ are the normalized maximum and minimum values of the $R, G$ and $B$ components. The computed values of $H, S$ and $V$ all lie in the range 0 to 1 , and are therefore converted to equivalent values in the range $0 \sim 255$ in order to enable a greater versatility in specifying suitable threshold values for each component.

Figure 1 illustrates the basic steps in the proposed imageprocessing scheme. As discussed above, the process commences by transforming the CCD image of the two targets from the RGB color space to the corresponding hue and saturation spaces (see Figs. 2(a) and 2(b), respectively). For computational convenience, the hue and saturation images are then converted into equivalent binary images by applying threshold values of $H_{\min }(60) / H_{\max }(85)$ and $S_{\min }(60) / S_{\max }(180)$, respectively (see Figs. 2(c) and 2(d)). Thereafter, the two images are multiplied to produce a composite H-S binary image (see Fig. 2(e)). Note that the 'AND' block shown in Fig. 1 is used to indicate that both conditions to the left of the 'AND' block must be satisfied simultaneously. A $3 * 3$ median filter is applied to remove any noise in the image, and a morphological closing operation is then performed to repair any resulting damage to the contours of the two targets (see Fig. 2(f)). Finally, the two targets in the image are detected by performing a blob analysis with a minimum threshold pixel value of 20 (see Fig. 2(g)). The blob analysis block is used to calculate the statistics associated with the images of the leading marks, such as the total number of pixels. Having acquired the two targets, their respective centers of gravity (CG) are computed in order to construct the leading line, leading marks location and the deviated heading angle.

\section{Visual Guidance Strategy}

As described in the Introduction, the autopilot system developed in this study is based on the leading line visual guidance strategy. Thus, as described in the sub-sections below, 


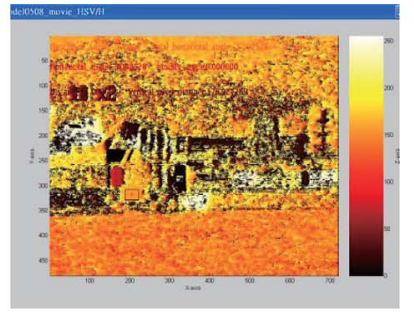

(a)

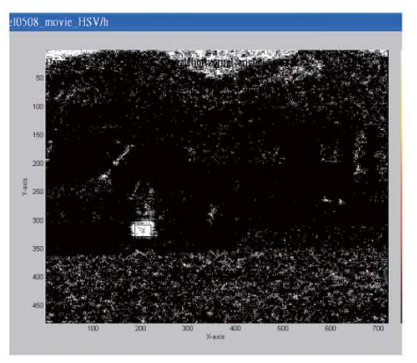

(c)

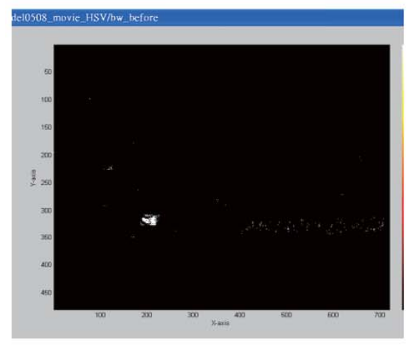

(e)

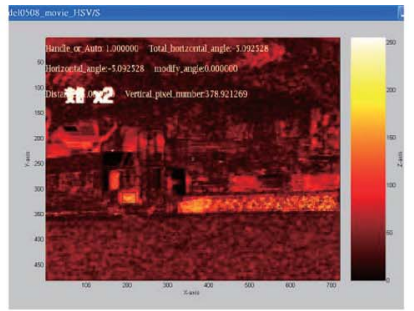

(b)

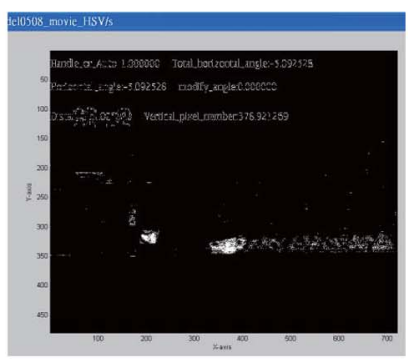

(d)

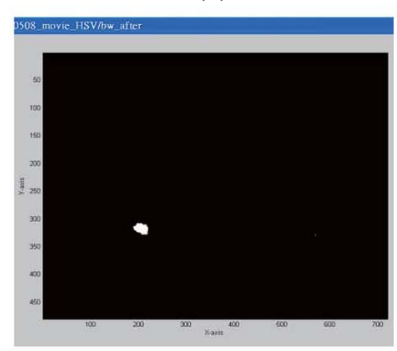

(f)

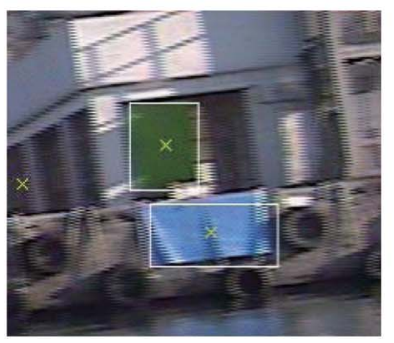

(g)

Fig. 2. (a) $H$-component image, (b) $S$-component image, (c) binary equivalent of $\boldsymbol{H}$-component image, (d) binary equivalent of $\mathrm{S}$-component image, (e) composite $\mathrm{H}$-S binary image, (f) filtered and repaired $\mathrm{H}-\mathrm{S}$ binary image, (g) acquisition of targets in image.

in mimicking the actions of a human pilot, the autopilot system requires a knowledge of: (1) the orientation of the ship relative to the front leading mark; (2) the position of the ship relative to the leading line; and (3) the distance between the ship and the berthing wall. This information is then used to instruct the necessary course adjustments required to bring the ship toward the leading line and to determine the appropriate moment at which to switch from an approach maneuvering mode to a berthing control mode.

\section{1) Image Coordinates}

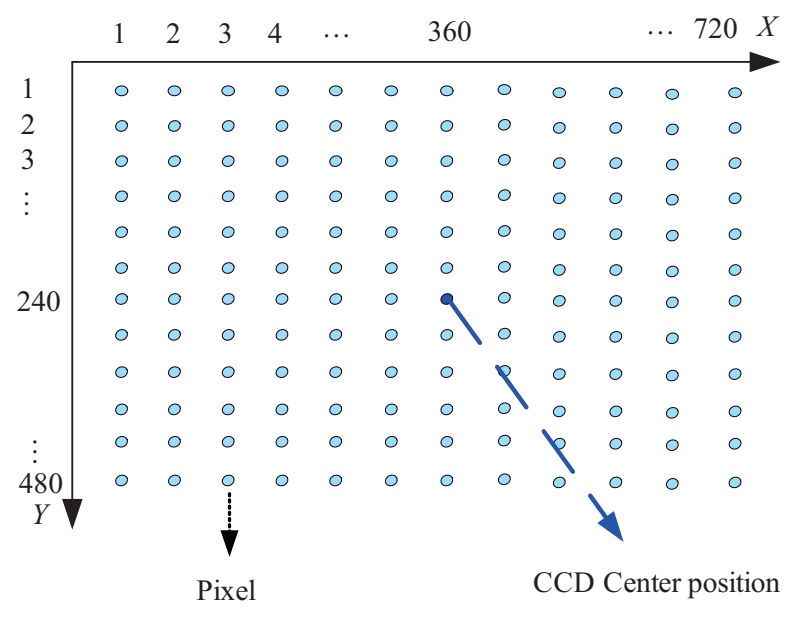

Fig. 3. Image coordinate framework.

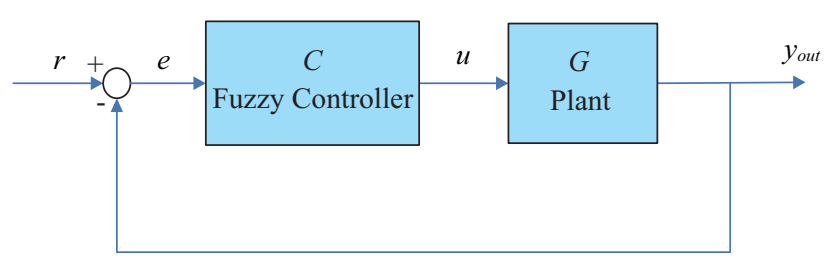

Fig. 4. Classical feedback control structure.

Figure 3 illustrates the image coordinate framework used by the autopilot system in computing the heading data of the ship and the distance of the ship from the berthing wall. The CCD camera used in the current trials had a resolution of $720 * 480$ pixels, i.e. the maximum $\mathrm{X}$-axis value in the image coordinate framework is 720 , while that of the Y-axis is 480 . As a result, the center of the CCD image is located at coordinates $(360,240)$.

According to the manufacturer's specification, the CCD camera has a horizontal field of view (HFOV) of $47.31^{\circ}$ and a vertical field of view (VFOV) of $36.32^{\circ}$. However, in the trials, the CCD camera was operated in a $2 \times$ zoom mode, and thus the HFOV and VFOV were reduced to $17.91^{\circ}$ and $13.76^{\circ}$, respectively.

\section{2) Computation of Deviated Heading Angle}

The structure of the autopilot system developed in this study is illustrated in Fig. 4, in which $G$ is the plant to be controlled, $C$ is the fuzzy controller, $r$ is the reference input signal, $e$ is the error signal, $u$ is the output command of the controller, and $y_{\text {out }}$ is the system output. As discussed below, the error signal in the current controller has the form of a "deviated heading angle", computed by the autopilot system using the CG information of the front and rear leading marks, respectively.

The deviated heading angle consists of two terms, namely $\theta_{1}$ and $\theta_{2}$, where $\theta_{1}$ describes the orientation of the ship relative to the berthing point and $\theta_{2}$ describes the position of the ship 


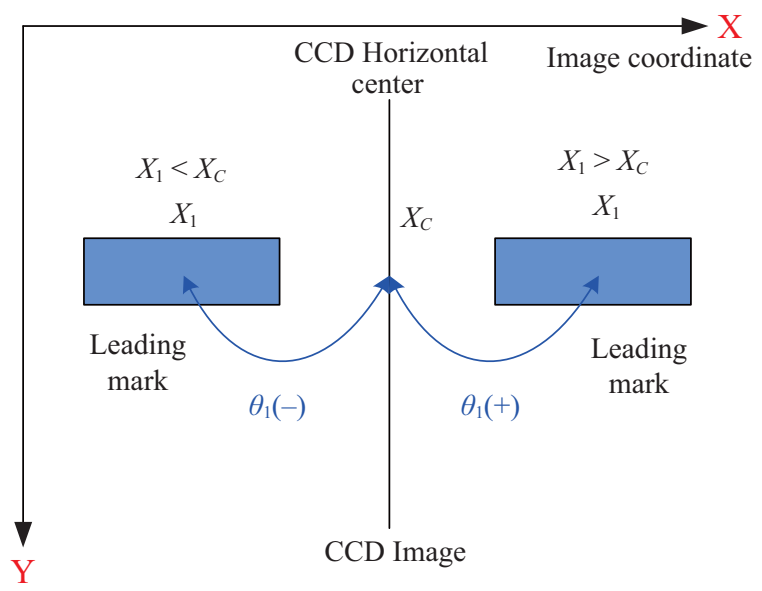

Fig. 5. Geometric relationship between $\theta_{1}$ and the front leading mark.

relative to the leading line. The first term, designated as the heading deviation angle, is derived from the displacement of the $\mathrm{CG}$ of the front leading mark from the center of the CCD camera screen. Since the center line of the CCD camera is aligned with the center line of the boat, the effect of $\theta_{1}$ is to point the bow of the boat at the front leading mark mounted on the berthing wall.

In Fig. 5, let $x_{1}$ denote the X-coordinate value of the CG of the front leading mark and let $x_{\mathrm{c}}$ denote the X-coordinate value of the center of the CCD camera screen. The heading deviation angle $\theta_{1}$ can then be computed as follows:

$$
\theta_{1}=\left(x_{1}-x_{c}\right) \times k_{x}
$$

where $k_{x}$ is defined as

$$
\begin{aligned}
k_{x} & =\frac{\mathrm{HFOV} \text { of CCD camera }}{\text { Total number of CCD pixels in horizonal direction }} \\
& =\frac{17.91}{720}=0.0248
\end{aligned}
$$

In other words, $k_{x}$ represents the HFOV angle of each image pixel in the X-direction.

The second term in the deviated heading angle, i.e. $\theta_{2}$ (designated as the tracking deviation angle), describes the position of the ship relative to the leading line, and varies as a function of the horizontal distance between the CGs of the front and rear leading marks in the CCD image, i.e.

$$
\theta_{2}=\left(x_{1}-x_{2}\right) \times k_{x}
$$

where $x_{1}$ and $x_{2}$ are the $\mathrm{X}$-coordinate values of the CGs of the front and rear leading marks, respectively, and $k_{x}$ is the calibration coefficient defined in (5).

The effect of the tracking deviation angle is illustrated

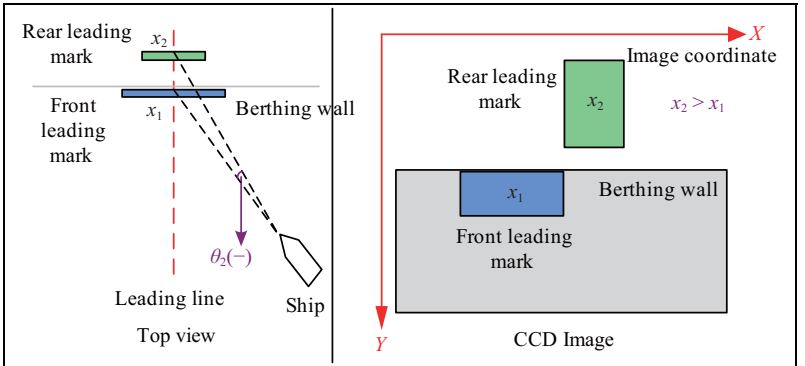

(a)

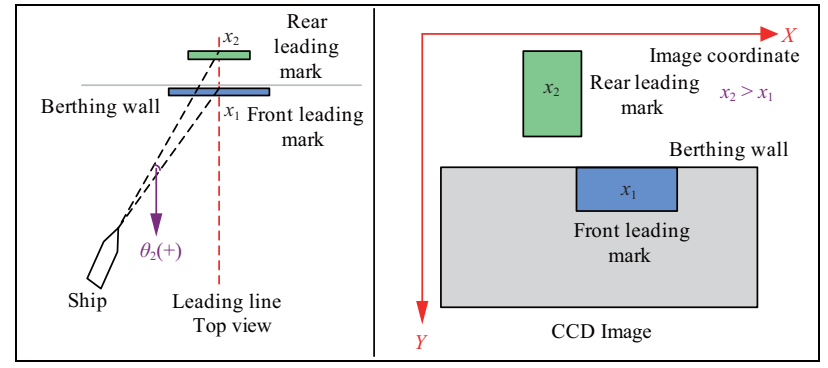

(b)

Fig. 6. (a) Ship located to right of leading line ( $\theta_{2}$ : Negative), (b) ship located to left of leading line $\left(\theta_{2}\right.$ : Positive).

schematically in Figs. 6(a) and 6(b). Note that depending on the location of the boat relative to the leading line, the rear(green) leading mark might appear to the right or left of the front (blue) leading mark during the approaching phase as shown in Figs. 6(a) and 6(b). In Fig. 6(a), the parallax effect causes the center of the rear leading mark to lie to the right of that of the front leading mark when viewed from the boat. Thus, $x_{2}$ is greater than $x_{1}$ in (6), and consequently a negative value of $\theta_{2}$ is produced. The negative sign of the tracking deviation angle is interpreted by the autopilot system as a sign that the ship is located to the right (starboard side) of the leading line and that a port helm movement is therefore required to steer the ship back on course. Figure 6(b) illustrates the opposite scenario, in which the ship is located to the left (port side) of the leading line. In this case, the rear leading mark lies to the left of the front leading mark, and thus (6) produces a positive value of $\theta_{2}$. Consequently, the autopilot system recognizes the need for a starboard helm command to steer the ship toward the leading line.

\section{3) Distance Estimation}

In the autopilot system developed in this study, the distance between the ship and the berthing wall is estimated using a simple trigonometric method. As shown in Fig. 7, the elevation angle $\theta_{y}$ of the rear leading mark is obtained as

$$
\theta_{y}=\left(y_{1}-y_{2}\right) \times k_{y}
$$

where $y_{1}$ and $y_{2}$ are the Y-coordinate values of the CGs of the front and rear leading marks, respectively, and $k_{y}$ is defined as 


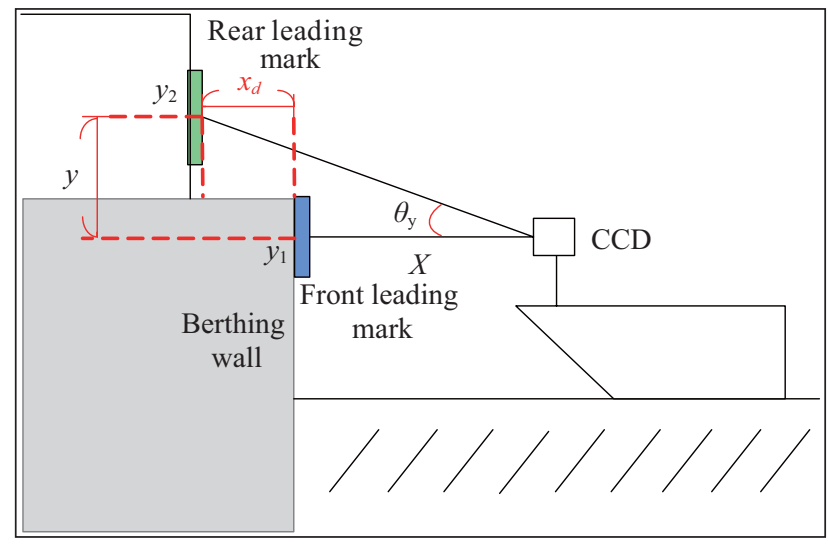

Fig. 7. Distance estimation based upon basic geometric principles.

$$
k_{y}=\frac{\text { VFOV of CCD camera }}{\text { Total number of CCD pixels in vertical direction }}
$$

$$
=\frac{13.76}{480}=0.0286
$$

In other words, $k_{y}$ represents the VFOV angle of each image pixel in the Y-direction.

Having computed the value of the elevation angle, the distance between the boat and the berthing wall is estimated simply in accordance with

$$
x=\frac{y}{\tan \theta_{y}}-x_{d}
$$

where $y$ is the vertical separation distance between the centers of the front and rear leading marks and is measured when arranging the targets on the quayside, and $x_{d}$ is the horizontal distance between the two targets and is also known in advance.

Although not specifically discussed in this study, the estimated distance between the ship and the berthing wall serves two purposes in the autopilot developed in this study, namely (1) to decide the appropriate moment at which to switch from an approach maneuvering mode to a berthing control mode, and (2) to provide a feedback error signal with which to fine-tune the steering commands during the berthing procedure at the quayside.

\section{FUZZY CONTROL SCHEME}

As discussed in previous section, the autopilot system developed in this study utilizes a fuzzy controller in which the error signal, $e$, is given by the heading deviation angle, $\theta_{1}$, and the tracking deviation angle, $\theta_{2}$. Furthermore, as discussed in relation to Figs. 6(a) and 6(b), a positive value of $\theta_{2}$ indicates that the ship is positioned to the port side of the leading line, while a negative value of $\theta_{2}$ indicates that the ship is positioned to the starboard side of the leading line. Finally, the
Table 1. Fuzzy control rules.

\begin{tabular}{|c|c|c|c|}
\hline & theta1_n & theta1_m & theta1_p \\
\hline theta2_n & n_verylarge & n_large & n_slightlarge \\
\hline theta2_m & n_morelarge & medium & p_morelarge \\
\hline theta2_p & p_slightlarge & p_large & p_verylarge \\
\hline
\end{tabular}

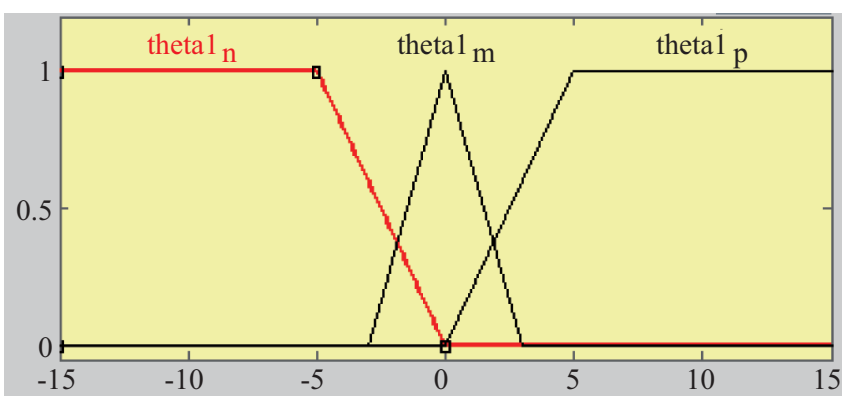

Fig. 8. Membership function of $\theta_{1}$.

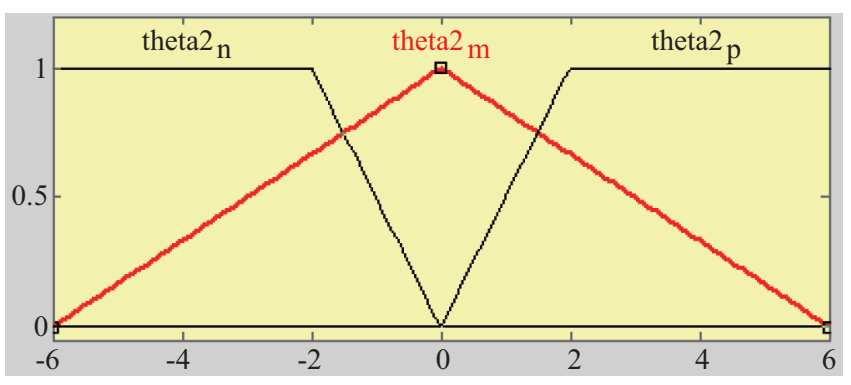

Fig. 9. Membership function of $\theta_{2}$ (type I).

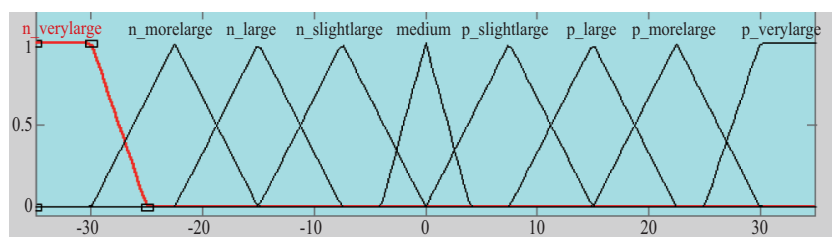

Fig. 10. Membership function of rudder command.

magnitude of $\theta_{2}$ indicates the distance by which the ship is offset to the left or the right of the leading line. Thus, given a knowledge of the signs and magnitudes of $\theta_{1}$ and $\theta_{2}$, the autopilot possesses sufficient information to orientate the bow of the boat toward the berthing point and to issue the rudder commands required to bring the ship back toward the leading line. In practice, however, it is impossible to relate $\theta_{1}, \theta_{2}$, and the rudder command via a direct mathematical relationship. Thus, in the present study, the necessary course changes are computed by a fuzzy controller based on the fuzzy rules shown in Table 1. Typical membership functions of $\theta_{1}$ and $\theta_{2}$ are shown in Figs. 8 and 9, respectively, while that of the output rudder command is shown in Fig. 10. The membership function of $\theta_{2}$ shown in Fig. 9 will be denoted by type I to differ- 


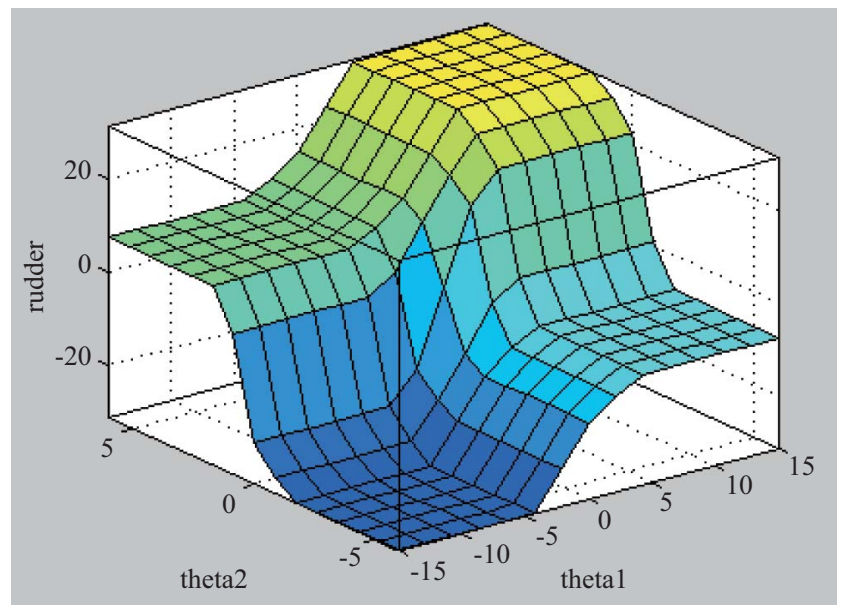

Fig. 11. I/O contour plot of fuzzy controller.

entiate it from the other type of membership function that appears in latter section.

Note that in Table 1, _n represents a negative value, _p represents a positive value and_m represents a near zero value. If we apply the fuzzy control rules with the case $\mathrm{A}_{1}$ condition in Fig. 6(a), the output response would be the p_slightlarge, this means a starboard helm command would be assigned to the controller. Meanwhile, in Fig. 10, a positive sign denotes a starboard command, a negative sign denotes a port command, and the value of the membership function indicates the rudder command angle. The overall input-output contour plot of the fuzzy controller is illustrated in Fig. 11.

\section{SYSTEM DESCRIPTION}

\section{Hardware Structure}

The performance of the fuzzy-based autopilot system was evaluated by performing a series of approach maneuvering trials using a four-meter FRP boat. As shown in Fig. 12, a CCD camera was installed at the bow of the boat and connected to a PC running the image-processing scheme and fuzzy autopilot system. The boat was equipped with two outboard motors, one mounted near the bow for berthing purposes, and the other mounted at the stern for propulsion and steering adjustment purposes. During the approach procedure, the distance of the boat from the berthing wall was estimated in accordance with (9) and was also computed directly for reference purposes by a differential global positioning system (DGPS). Finally, the heading deviation angles and tracking deviation angles were computed by the image-processing scheme.

\section{Software Structure}

As shown in Fig. 13, the software structure was implemented using two PCs, namely a host PC (PC1) and a target PC (PC2). PC1 was interfaced with the CCD via a TV card and executed the image-processing and fuzzy controller schemes utilizing Matlab simulation software with the Simulink

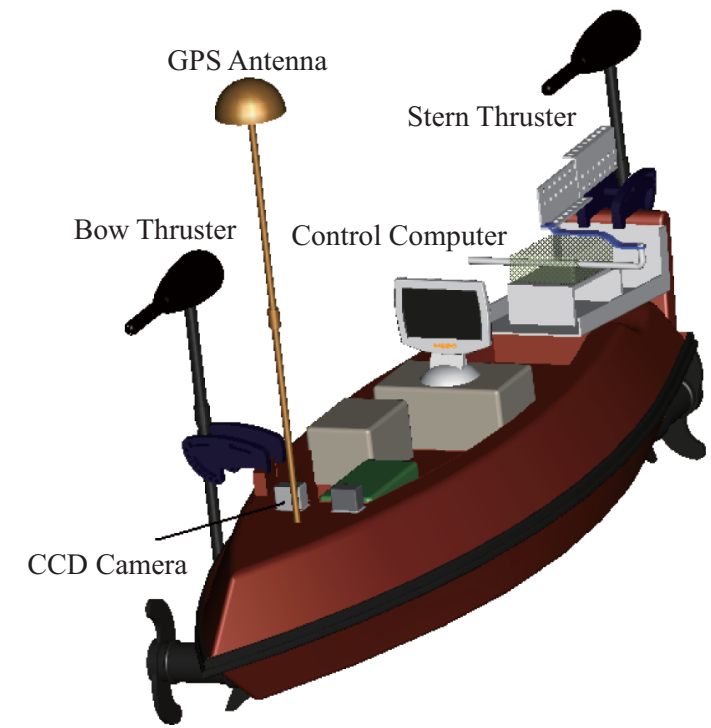

Fig. 12. Test bed installed on FRP boat used for autopilot evaluation trials.

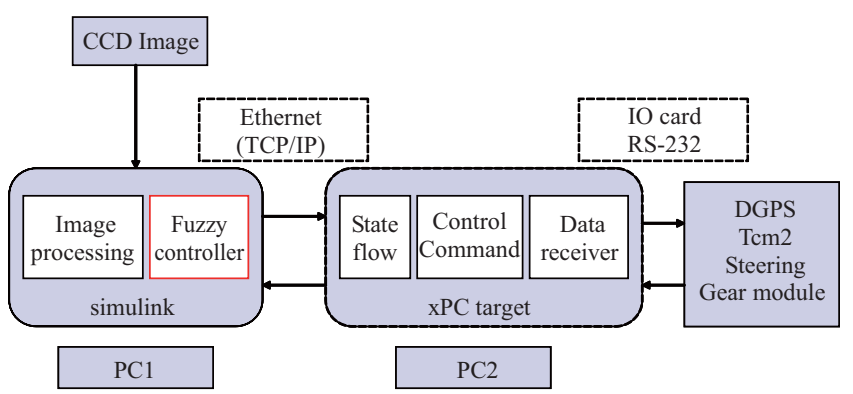

Fig. 13. Software structure implemented on host PC and xPC Target PC.

Video and Image Processing Blockset [10]. Meanwhile, PC2, running in the XPC Target environment [11], was fitted with various $\mathrm{A} / \mathrm{D}, \mathrm{D} / \mathrm{A}$ and $\mathrm{I} / \mathrm{O}$ cards and was used to instruct the steering module of the FRP boat in line with the rudder command signals generated by the fuzzy controller installed on the host PC. The time between the image being acquired by $\mathrm{PC} 1$ and the corresponding control command being issued by PC2 was found to be approximately $0.67 \mathrm{sec}$. Hence, the image processing rate was of the order of $1.5 \mathrm{~Hz}$.

\section{EXPERIMENTAL TRIALS AND RESULTS}

The experimental trials were performed in the harbor attached to National Taiwan Ocean University in northeast Taiwan. As shown in Fig. 14, the boat was positioned initially to the left of the leading line at a distance of approximately 85 $\mathrm{m}$ from the berth and $60 \mathrm{~m}$ from the designated berthing standby zone. The experiment started by manually steering the boat until the two targets were acquired by the CCD camera. At this point, the autopilot was set to its automatic approach mode and was used to guide the boat automatically 


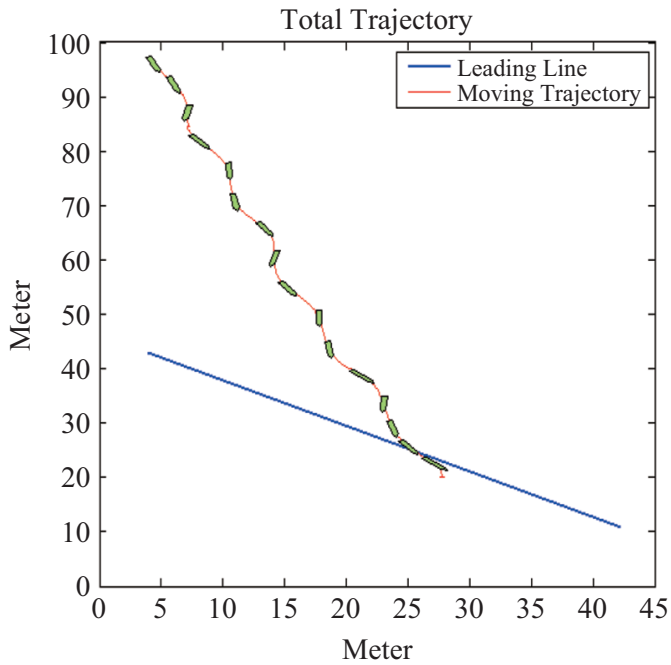

(a)

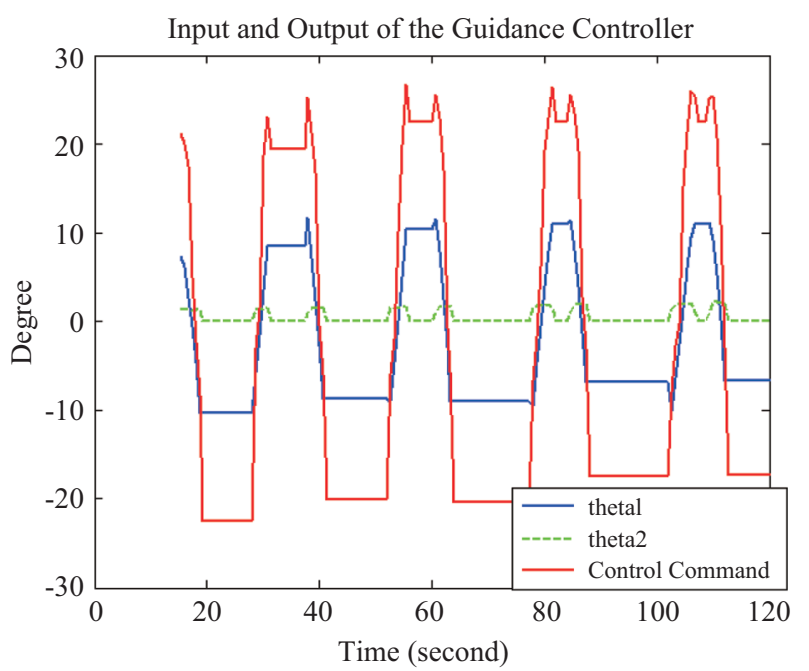

(b)

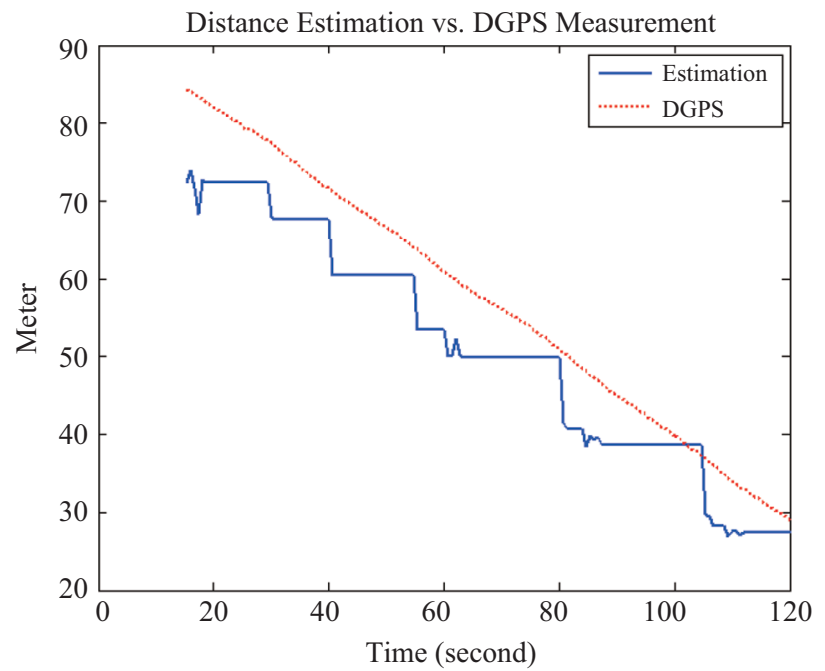

(c)

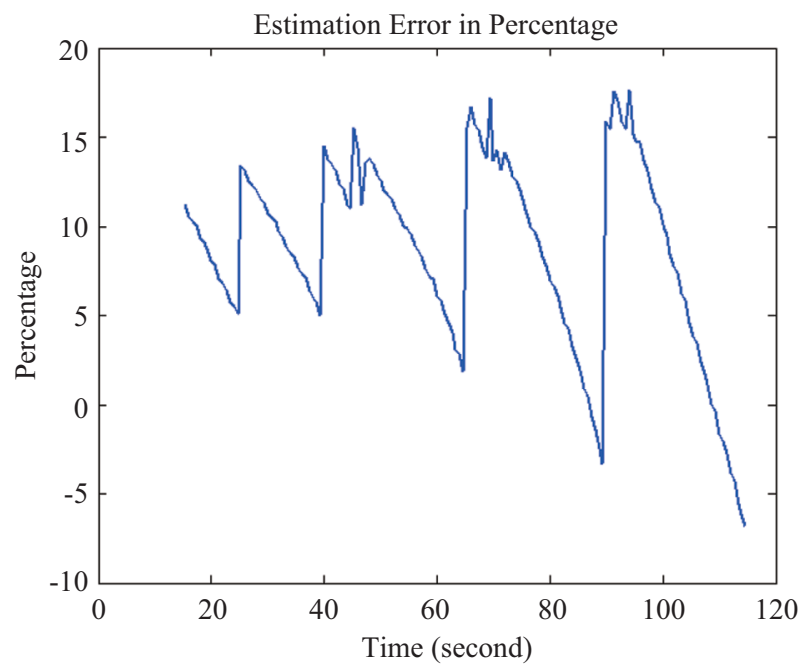

(d)

Fig. 15. (a) Experimental trajectory, (b) time-dependent variation of inputs and outputs of fuzzy-based autopilot system, (c) comparison of estimated and exact results for ship-to-quay distance, (d) time-varying error percentage between estimated and exact ship-to-quay distance.

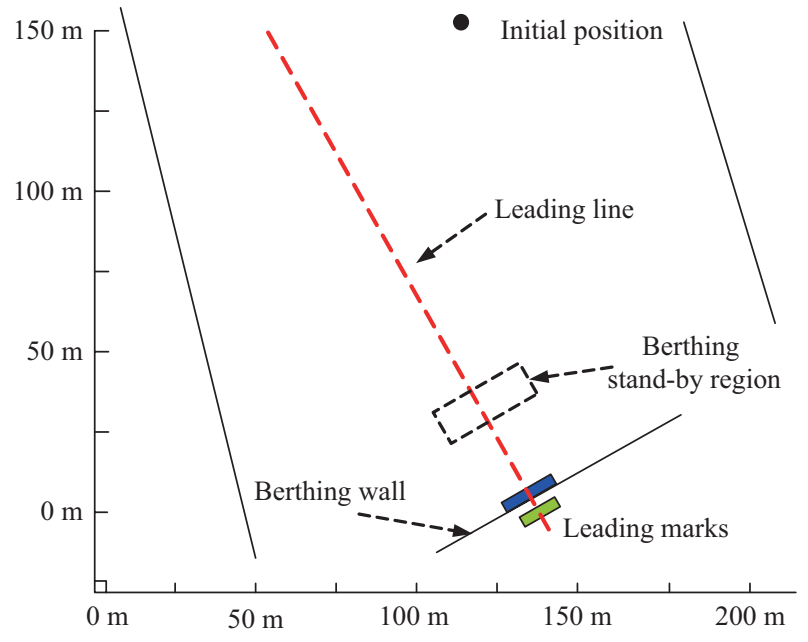

Fig. 14. Experimental arrangement. toward the leading line. The experiment continued until the boat entered the berthing stand-by zone, at which point the autopilot system switched automatically from the approach mode to the berthing control mode. (Note that in this study, the berthing capabilities of the autopilot were not evaluated, and thus the experiments terminated as soon as the system switched into the berthing control mode.)

Figure 15(a) shows the trajectory of the FRP boat relative to the leading line during the automatically-controlled approaching maneuver when the membership functions defined by Figs. 8-10 are used. The results clearly show that the boat converges toward the leading line as it approaches the berthing stand-by zone. Therefore, the efficacy of the fuzzy-based autopilot system in accomplishing the approaching maneuver is confirmed. Figure 15(b) illustrates the variations in the input and output variables of the fuzzy controller during the approaching maneuver. It is observed that the tracking devia- 


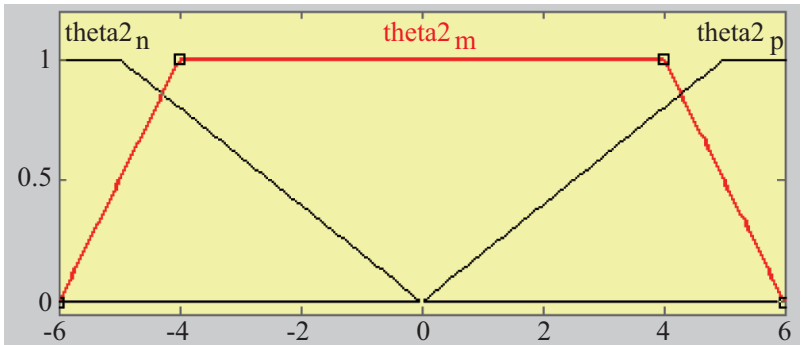

(a)

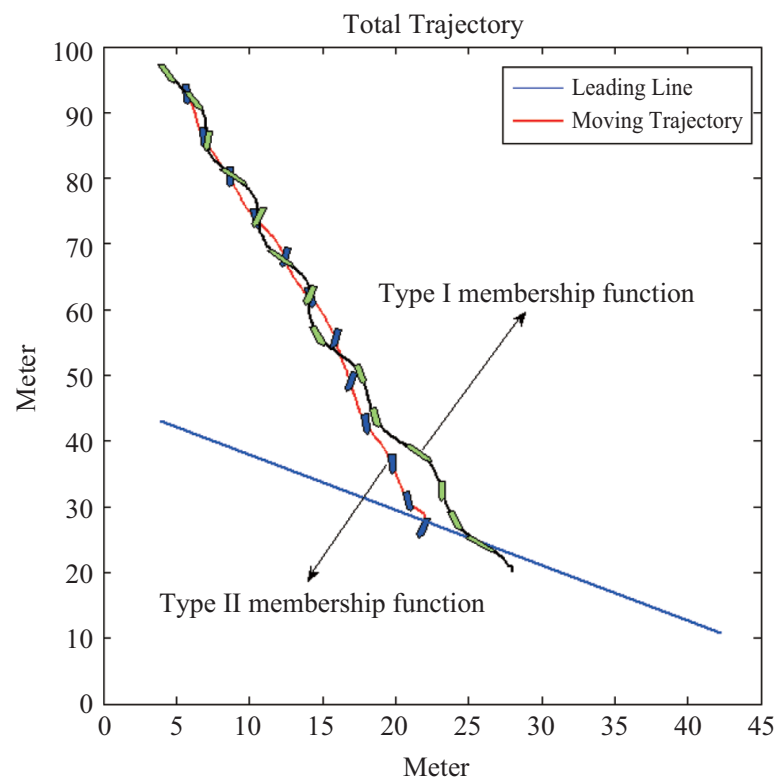

(b)

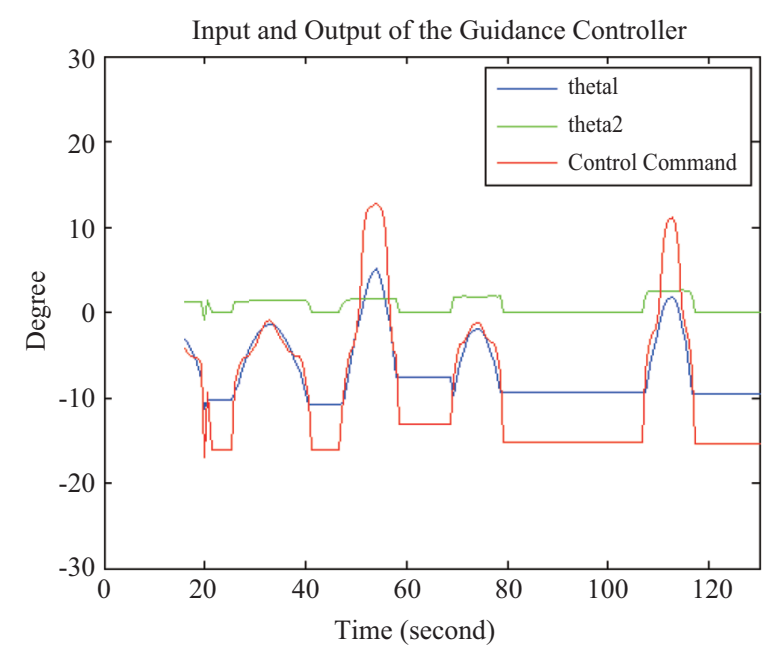

(c)

Fig. 16. (a) Membership function of $\theta_{2}$ (type II), (b) comparison of experimental trajectories for type I, and II membership functions of $\theta_{2}$, (c) time-dependent variation of inputs and outputs of fuzzy-based autopilot system.

tion angle, $\theta_{2}$, maintains a relatively low value throughout. This result is the consequence of the small separation distance between the front and rear targets on the berthing wall (i.e. $\left.x_{d}=2.7 \mathrm{~m}\right)$. Note that a larger separation distance, whilst desirable, was impossible in the current evaluation trials due to the limited space available at the quayside. Note also that in Fig. 15(b), the profiles level-off after approximately $t=120$ seconds since at this point, the boat entered the berthing stand-by zone, and thus the experimental measurements were discontinued. Figure 15(c) illustrates the time-varying distance of the ship to the berthing wall as estimated by the image-processing scheme (solid line) and measured by the DGPS system (dotted line), respectively. Although the estimated results are in broad qualitative agreement with the exact results, it is clear that quite significant errors exist. The time-varying discrepancy between the two sets of results is illustrated graphically in Fig. 15(d). From inspection, the error in the estimated distance value is found to vary in the range of approximately 10 20\%. In addition, it is observed that the magnitude of the error tends to increase as the boat approaches the berthing wall. This result is to be expected since the rear target elevation angle $\theta_{y}$ increases significantly as the ship nears the quayside, and thus the value of the ship-to-berth separation distance computed using (9) becomes increasingly sensitive to errors in its estimated value. The image-processing scheme is designed such that in the event that the targets disappear from the CCD screen (as the result of course changes, for example), the previous estimate of the shipto-berth distance is retained as the current estimated value. Thus, as shown in Fig. 15(c), the time-varying estimated distance profile has a pronounced stair-like characteristic.

Since the FRP boat used in the current experiments is small (i.e. $4 \mathrm{~m}$ in length) and therefore highly responsive, large rudder commands result in an abrupt change of course and cause the CCD camera to lose track of the leading marks. Thus, unless suitable precautions are taken, the imageprocessing scheme can not compute feasible values of the input variables $\theta_{1}$ and $\theta_{2}$. To resolve this problem, the autopilot system was designed to automatically adopt the following values of $\theta_{1}$ and $\theta_{2}$ whenever the leading marks were lost from the CCD image:

$$
\begin{gathered}
\theta_{1}(t)=\theta_{1}(t-1) \\
\theta_{2}(t)=0
\end{gathered}
$$

where $t$ indicates the present time step and $t-1$ indicates the previous time step. From the definition of the tracking deviation angle $\theta_{2}$ in (6), it can be seen that a value of $\theta_{2}=0$ implies that the boat is aligned along the leading line. Thus, the autopilot system considers only the effect of the heading deviation angle, $\theta_{1}$. Since the function of $\theta_{1}$ is to point the bow of the boat at the front leading mark, the effect of (10)-(11) is therefore to turn the boat in the direction of the front target. As a result, the CCD camera re-acquires the two targets, and thus the ability of the image-processing scheme to compute feasible values of $\theta_{1}$ and $\theta_{2}$ is restored. 


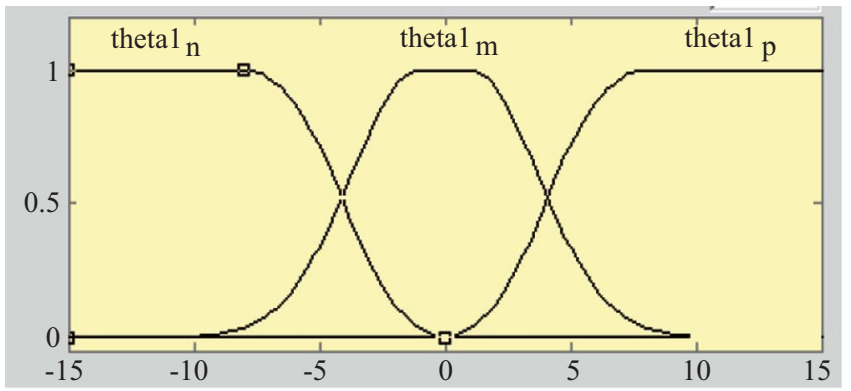

(a)

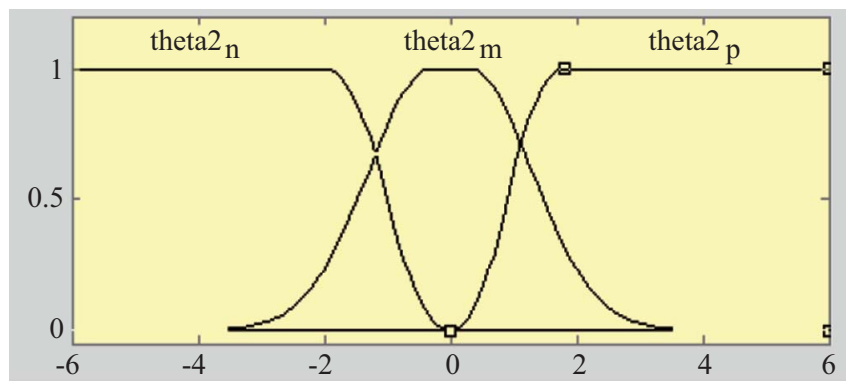

(b)

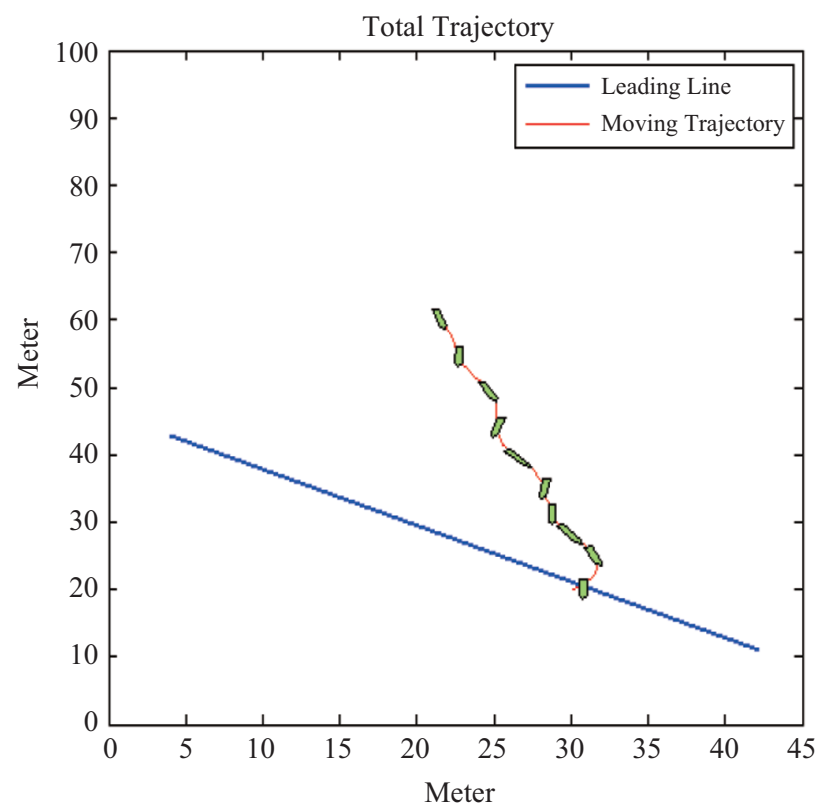

(c)

Fig. 17. (a) The bell-shaped membership function of $\theta_{1}$, (b) the bellshaped membership function of $\theta_{2}$, (c) experimental trajectory.

It seems logical to infer that the tracking deviation angle $\theta_{2}$ plays a far more important role than the heading deviation angle $\theta_{1}$ in the proposed leading line guidance strategy because the separation distance of the leading marks was used in computing the angle $\theta_{2}$ (see (6)). Hence, it would be interesting to see the effect caused by a change in the $\theta_{2}$ membership function alone, while keeping the others unchanged. Therefore, a different type (type II) of membership function for $\theta_{2}$ was selected (see Fig. 16(a)), while the $\theta_{1}$ and the rudder command membership functions were the same as those given in Figs. 8 and 10 respectively.

Specifically, Fig. 16(b) shows simultaneously the trajectories resulted from the fuzzy autopilot system using type I (Fig. 9) and type II (Fig. 16(a)) membership functions of $\theta_{2}$. It is observed that the trajectory is less oscillatory and the boat appears to head directly for the front leading mark, instead of converging toward the leading line. Figure 16(c) illustrates the variations in the input and output variables of the fuzzy controller type II membership function for $\theta_{2}$ during the approaching maneuver. It is found that the control commands issued by the type II fuzzy controller are much less excessive when compared with those of type I fuzzy controller given in Fig. 15(b). Hence, a less oscillatory trajectory is produced when type II membership function for $\theta_{2}$ has been adopted.

The main difference between the $\theta_{2}$ membership functions given in Figs. 9 and 16(a) is the definition of the near-zero state. Specifically, in Fig. 16(a), if $\left|\theta_{2}\right| \leq 4^{\circ}$, then the state is considered to be near-zero. However, Fig. 9 adopts a tighter criterion; namely, the near-zero state is reached only when $\theta_{2}$ is exactly zero. Owing to a small separation distance between the leading marks in our experimental setup, the computed $\theta_{2}$ angle rarely exceeds $2 \mathrm{deg}$. Hence, with the type II membership function of Fig. 16(a), the $\theta_{2}$ state is almost always considered to be near-zero, and the autopilot thinks the boat is already on the leading line. Therefore, no attempt is made to steer the boat toward the leading line, and the boat heads directly for the front leading mark placed on the berth.

However, with the membership function given in Fig. 9, even for a small value of $\theta_{2}$, the boat is considered to be off the leading line. Hence, the heading of the boat is constantly varied in order to steer the boat toward the leading line. This partly explains the oscillatory nature of the trajectory shown in Fig. 15(a).

Finally, smooth bell-shaped membership functions of $\theta_{1}$ and $\theta_{2}$ as shown in Figs. 17(a) and 17(b) respectively were used in the experiments. Moreover, the range defines the nearzero state of the tracking deviation angle $\theta_{2}$ is larger than that of Fig. 9, but smaller than that of Fig. 16(a). The resulting trajectory is given in Fig. 17(c), and the trajectory seems to be a mixture of the trajectories shown in Figs. 15(a) and 16(b). Specifically, in terms of the degree of trajectory oscillation, the trajectory shown in Fig. 17(c) is smaller than that of Fig. 15(a), but larger than that of Fig. 16(b). However, in terms of trajectory convergence to the leading line, it is worse than that of Fig. 15(a), but better than that of Fig. 16(b).

Therefore, a trade-off between the trajectory convergence to the leading line and the degree of trajectory oscillation can be achieved through proper selection of the types of membership functions in the experiments.

\section{CONCLUSIONS}

This paper has developed an autopilot system which mimics the behavior of a human pilot in accomplishing the 
approaching maneuver of a small boat in a harbor using the leading line visual guidance method. In the proposed approach, two targets are arranged in cascade on the quayside and are detected by a CCD camera mounted on the bow of the boat. An image-processing algorithm is used to compute the heading deviation angle and tracking deviation angle of the boat with respect to the leading line passing through the centers of gravity of the two targets. The two deviation angles are then supplied to a fuzzy-rules-based controller which computes the course adjustments required to bring the ship toward the leading line and then issues the corresponding rudder instructions. The experimental results performed using a small FRP boat in a real-world harbor have confirmed the ability of the proposed autopilot system to guide the boat in such a way that it converges toward the leading line and enters the designated pre-berthing region. To enable the autopilot system to determine the appropriate point at which to switch from an approach maneuver mode to a berthing control mode, a method has been proposed for computing the distance between the ship and the quay based on the perceived elevation angle of the rear target and the known separations of the front and rear targets in the horizontal and vertical directions, respectively. The experimental results have shown that the estimated value of the ship-to-quay distance deviates by around 10 20\% from the exact value. Whilst this result confirms the general feasibility of using the proposed range estimation scheme to determine the point at which to switch from an approach mode to a berthing control mode, it is clear that further work is required to improve the accuracy of the distance estimates such that they can be used for feedback purposes during the actual berthing procedure. This issue is presently under review by the current group and will be presented in the near future.

\section{ACKNOWLEDGMENTS}

This study was supported by the National Science Council of the Republic of China under Grant No. NSC-95-2221-E-
019-074-MY3.

\section{REFERENCES}

1. Chao, C. H., "Omni-directional vision-based parallel-parking control design for car-like mobile robot," Proceedings of the 2005 IEEE International Conference Mechatronics, Taiwan, pp. 562-567 (2005).

2. Holzhüter, T., "LQG approach for the high- precision track control of ships," IEE Proceedings- Control Theory Applications, Vol. 144, No. 2, pp. 121-127 (1997).

3. Huang, C. C., Application of the Sliding Mode Fuzzy Logic Controller to the Guidance and Control of an Autonomous Underwater Vehicle, Master's thesis, Department of Engineering Science and Ocean Engineering, National Taiwan University, Taipei, Taiwan (1999) (in Chinese).

4. Lee, S. D., Tzeng, C. Y., Huang, B. S., Huang, C. G., Kang, C. K., and Kehr, Y. Z., "Application of image processing to the control of a small boat," Journal of Taiwan Society of Naval Architects and Marine Engineers, Vol. 27, No. 3, pp. 103-112 (2008) (in Chinese).

5. Minorsky, N., "Directional stability of automatically steered bodies," Journal of the American Society of Naval Engineers, Vol. 34, No. 2, pp. 280-309 (1922).

6. Morawski, L. and Pomirski, J., "Ship track-keeping: Experiments with a physical tanker model," Control Engineering Practice, Vol. 6, No. 6, pp. 763-769 (1998).

7. Proctor, A. A., Johnson, E. N., and Apker, T. B., "Vision-only control and guidance for aircraft," Journal of Field Robotics, Vol. 23, No. 10, pp. 863-890 (2006).

8. Sperry, E., "Automatic steering," Transactions of the Society of Naval Architects and Marine Engineers, Vol. 30, pp. 53-61 (1922).

9. Suzuki, Y., "Parking assistance using multi-camera infrastructure," Proceedings of the 2005 IEEE Intelligent Vehicles Symposium, Las Vegas, Nevada, pp. 106-111 June (2005).

10. The Math Works Inc, Video and Image Processing Blockset ${ }^{T M}$ Reference, Version 2.3 (Release 2007a).

11. The Math Works Inc, xPC Target, Version. 3 (2006).

12. Tzeng, C. Y. and Lu, G. H., "An internal model control- based neural network ship steering autopilot design," Journal of the Society of Naval Architects and Marine Engineers of the ROC, Vol. 22, No. 1, pp. 13-23 (2003) (in Chinese).

13. Vaneck, T. W., "Fuzzy guidance controller for an autonomous boat," IEEE Control Systems Magazine, April, Vol. 17, No. 2, pp. 43-51 (1997).

14. Zhang, Y., Hearn, G. E., and Sen, P., "A multivariable neural controller for automatic ship berthing," IEEE Control Systems Magazine, Vol. 17, No. 4, pp. 31-45 (1997). 